\title{
Thermally and Anionically Initiated Cure of Bismaleimide Monomers
}

\author{
A. SERIS, M. FEVE, F. MECHIN, and J. P. PASCAULT*
}

Laboratoire des Matériaux Macromoléculaires, URA CNRS n 507, Institut National des Sciences Appliquées de Lyon, 20, Avenue A. Einstein, 69621 Villeurbanne Cedex, France

\section{SYNOPSIS}

A kinetic and thermodynamic study of the thermally or anionically induced cure of aromatic bismaleimide monomers (namely 2,4-bismaleimidotoluene, 4,4'-bismaleimidodiphenylmethane and their eutectic mixture) is presented. Calorimetric investigations allowed the determination of heats of polymerization and activation energies for both types of initiations, and the values found are compared with data in the literature. Isothermal cures were also carried out with and without anionic initiator (typically imidazole). SEC measurements were used to monitor the conversion of the monomers as a function of time. The thermally induced polymerization seems to proceed in a rather heterogeneous way, owing to an initiation that is too slow: gelation appears at first only as the formation of microgels, with no immediate increase in the viscosity; macrogelation occurs some time after. On the contrary, the anionically induced polymerization is more homogeneous. A complete study of a particular reacting system revealed no interference between radical and anionic polymerization over a wide range of temperatures, and in this case gelation results in an immediate increase in viscosity. However, the conversion at the gel point is unexpectedly high, which can be interpreted as the result of a very fast initiation as well as of transfer reactions. Glass transition temperatures were also measured as a function of time and curing temperature in order to study the influence of vitrification on the kinetics and to check for the existence of a unique relation between $T_{g}$ and conversion. This actually proved to be correct as long as diffusion does not slow down the reaction; in this case Gillham's treatment of $T_{g}$ versus Int was successfully applied and led to the correct value of the activation energy. All the measurements allowed us to establish the time-temperature-transformation cure diagram for a specific reacting system.

Published in Journal of Applied Polymer Science vol. 48, 257-269 (1993). 


\section{INTRODUCTION}

Thermosetting polyimides are of low-molecular weight, especially difunctional monomers, or prepolymers or mixtures thereof, which carry imide groups in their backbone structure and are terminated by reactive groups such as maleimide. Bismaleimide monomers are generally cured by a thermally induced addition reaction to give highly crosslinked systems. The final networks are good heat-resistant polymers and suitable for use in a wide range of materials. In particular they have been utilized in multilayer printed wiring boards for large-scale computers and in carbon fiber composites for the aerospace industry.

However, when bismaleimides are thermally cured, the high temperature needed for the beginning of the reaction and to fully cure the material limits their use in conventional composite processing. The use of initiators can effectively reduce the cure temperature. The initiators can be peroxides, ${ }^{1-4}$ resulting in a radical initiation, or they can be tertiary amines or imidazoles, ${ }^{5}$ resulting in an anionic initiation.

The purpose of this work is to evaluate the influence of different amounts of imidazole initiator on the reaction rate and on the phase transition process of a mixture of two bismaleimide monomers. During the curing of thermosets there are two kinds of phase transitions: gelation and vitrification. Gelation corresponds to the time when a giant tridimensional macromolecule is formed and the weight average molecular mass, $\overline{M_{w}}$, tends to infinity, because of crosslinking reactions. Vitrification occurs when the length of the macromolecules or the crosslink density increases such that the glass transition temperature, $T_{g}$, is the same as the curing temperature, $T_{i}$. Once $T_{g}$ has reached $T_{i}$, the system gets very viscous and the reactions become diffusion controlled. Reaction kinetics and physical transformation of a network are thus strongly interdependent.

This kind of study is an important step for process optimization and for a better understanding of structure-property relations in the final network.

\section{EXPERIMENTAL}

All the experiments were carried out using pure monomers or an eutectic mixture of 4,4'bismaleimidodiphenylmethane (BDM, $70 \%$ by weight) and 2,4-bismaleimidotoluene (BT, $30 \%$ by weight). The melting point of this eutectic mixture is $143^{\circ} \mathrm{C}$. $\mathrm{N}$-phenylmaleimide ( $\mathrm{N}$-PMI ) was used as a monofunctional model compound. The monomers were kindly provided to us by Rhône-Poulenc, and their purities were determined by proton nuclear magnetic resonance (NMR) by comparing the integration of the maleimide proton peak to those relative to the central moiety. The only impurity was found to be an acetoxy-derivative resulting from the addition of two acetic acid molecules on the BMI double bonds. Imidazole and benzimidazole (Aldrich) were used as catalysts without further purification. 

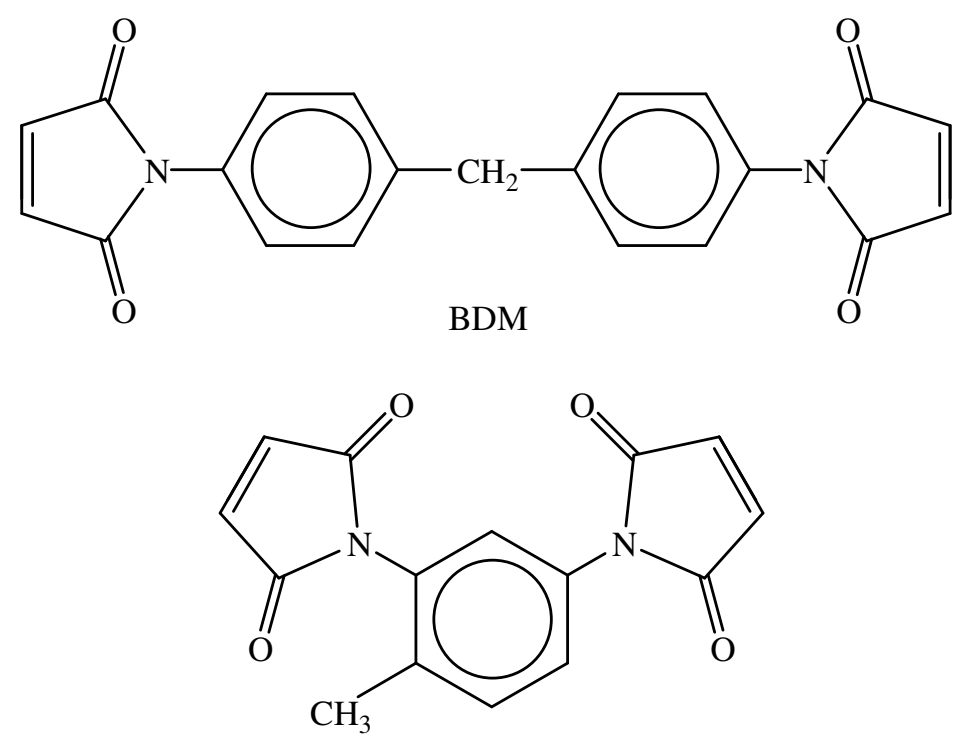

BT

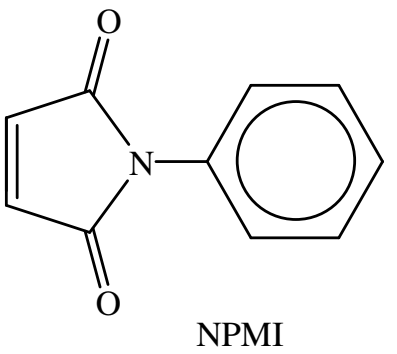

Preliminary investigations of the reactions were performed using differential scanning calorimetry (DSC) on a Mettler TA 3000 calorimeter. Thermograms were obtained for 5-10 mg samples using heating rates of $10 \mathrm{~K} / \mathrm{min}$ for reaction enthalpy measurements and $7.5 \mathrm{~K} / \mathrm{min}$ for glass transition temperature $\left(T_{g}\right)$ determinations (by convention, always taken as the onset value).

The isothermal kinetics of these reactions were then followed by size exclusion chromatography (SEC) performed with a Waters apparatus equipped with a differential refractometer and a UV absorption detector. The eluent was either tetrahydrofurane (THF) at room temperature or dimethylformamide (DMF) at $80^{\circ} \mathrm{C}$ for poorly soluble samples. Calibrations curves were obtained from polystyrene standards.

The viscosity during isothermal cures was monitored with a coaxial cylinder viscometer using shear rates equal to $100 \mathrm{~s}^{-1}$ or $0.65 \mathrm{~s}^{-1}$ on a Contraves Rheomat 115 .

Viscoelastic analysis as a function of time was performed with a RDA2 Rheometrics viscoelasticimeter, by stressing the reacting mixtures, situated between two parallel circular plates, at a frequency equal to $100 \mathrm{rad} / \mathrm{s}$.

In order to monitor the double-bond conversion during cure, a chemical determination of the remaining maleimide groups was sometimes used. For this purpose, the sample was dissolved or suspended in $\mathrm{N}$-methylpyrrolidone plus a small amount of aqueous acetic acid. The double bonds were then reacted with a known excess of morpholine in acetone to yield quantitatively the aspartimide adduct. The residual amine was finally titrated with hydrochloric acid. 


\section{RESULTS AND DISCUSSION}

\section{Kinetics Investigations from DSC Scans}

Maleimides are generally crystalline monomers. However, the two bismaleimides (BMI) used in this study can be obtained in the amorphous state with a glass transition temperature, $T_{g o}$, around $46-47^{\circ} \mathrm{C}$. But it is difficult to keep the samples amorphous because the crystallization rate is very high, even at room temperature. So during a DSC scan of a monomer sample to $380^{\circ} \mathrm{C}$ at $10 \mathrm{~K} / \mathrm{min}$, we usually observe first an endothermic peak corresponding to the melting temperature $T_{m}$, followed by an exothermic peak which represents the reaction of the double bonds, characterized by the peak area, $\Delta H \mathrm{~J} / \mathrm{g}$, and the temperature at the maximum, $T_{p}$. Typical DSC scans are shown in Figure 1.

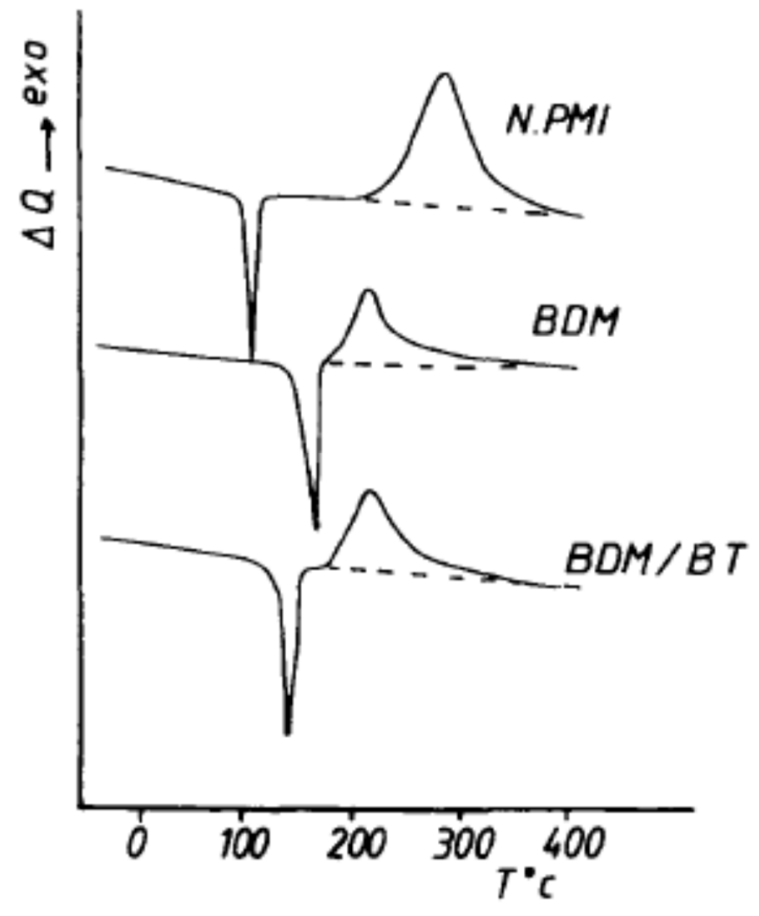

Figure 1. DSC thermograms of monofunctional (N-PMI) and difunctional monomers (BDM). Heating rate $q=10 \mathrm{~K} / \mathrm{min}$.

DSC has also been used with some assumptions by different workers ${ }^{6-8}$ to study the kinetics of thermoset curing reactions without attempting to determine the reaction order. The relation between the heating rate, $q$, and the peak exotherm temperature, $T_{p}$, is of the form:

$$
L n q=-\frac{E_{a}}{R T}+B
$$

where $q$ is the heating rate $(\mathrm{K} / \mathrm{min}), T_{p}$ is the absolute peak exotherm temperature, $B$ is a constant related to an Arrhenius frequency factor, and $R$ is the gas constant. The activation energy $E_{a}$ can be obtained from the slope of the plot of $\operatorname{Ln} q$ versus $1 / T_{p}$. Dynamic scans were run at $q=2.5,5,7.5$, and $10 \mathrm{~K} / \mathrm{min}$.

The chain polymerization of bismaleimide monomers may result from a chemical initiation if some initiator has been added (bases like alkoxides or imidazoles for anionic polymerizations, or peroxides for radical polymerizations) but it can also be thermally initiated simply by heating the mixture. In order 
to try and separate the different mechanisms which could be involved in the curing of bismaleimides, DSC scans were run at different heating rates on the monomers with and without anionic initiator.

Table I. Thermally induced polymerization of maleimide and bismaleimides

\begin{tabular}{|c|c|c|c|c|c|c|}
\hline Monomers (-Ar-) & $\begin{array}{c}\text { Purity } \\
(\%)\end{array}$ & $T_{g}\left({ }^{\circ} \mathrm{C}\right)$ & $T_{m}\left({ }^{\circ} \mathrm{C}\right)$ & $T_{p}{ }^{\mathrm{b}}\left({ }^{\circ} \mathrm{C}\right)$ & $\begin{array}{c}\Delta H_{p}^{\mathrm{b}, \mathrm{c}} \\
(\mathbf{k J} / \mathbf{m o l})\end{array}$ & $\begin{array}{c}E_{a} \\
(\mathbf{k J} / \mathbf{m o l})\end{array}$ \\
\hline maleimide & 99.9 & - & 90 & 276 & 85 & - \\
\hline$(\mathrm{N}-\mathrm{PMI})$ & 95 & 46 & 160 & 214 & 44 & 288 \\
\hline bismaleimide & 99.6 & 46 & 163 & 240 & 43 & 123 \\
\hline
\end{tabular}

${ }^{\text {a }}$ Checked by ${ }^{1} \mathrm{H}-\mathrm{NMR}$.

${ }^{b}$ Exotherm maximum $\left(T_{p}\right)$ and heat of polymerization $\left(\Delta H_{p}\right)$ are determined from DSC measurements using the heating rate $\mathrm{q}=10 \mathrm{~K} \cdot \mathrm{min}^{-1}$, and activation energy are determined using the heating rates $\mathrm{q}=2.5,5,7.5$ and $10 \mathrm{~K} \mathrm{~min}^{-1}$.

${ }^{\mathrm{c}}$ Relative to one double bond in the case of bismaleimide.

The thermally induced polymerization of the monomaleimide (Ar-MI) and two bismaleimides (MI-ArMI) was first examined. The results are reported in Table I. Polymerization occurs just after the melting temperature in the case of the two BMI's and at higher temperature in the case of N-PMI (Fig. 1). Several main features appear:

1. Similar results for $T_{p}$ and $E_{a}$ have been reported in the literature. ${ }^{1,2,4,9}$

2. In the case of the BMI, BDM, the presence of impurities even in small quantities enhances tremendously the activation energy (cf. Table I) and shifts the polymerization exotherm to lower temperatures (it also lowers the gelation time and promotes the overall kinetics-results not presented here). This behavior is in agreement with Brown's ESR studies on the radical polymerization of BDM which demonstrated the possible interference of impurities in the mechanism. ${ }^{10}$

3. Joshi and Zwolinski ${ }^{11}$ have reported an enthalpy of polymerization, $\Delta H_{p}=87 \mathrm{~kJ} / \mathrm{mol}$ for the monofunctional monomer $N$-PMI. We obtained a similar value $\Delta H_{p}=85 \mathrm{~kJ} / \mathrm{mol}$. This 
value for $N$-PMI is the same as the one reported in the literature for the thermal polymerization of ethylene.

4. But in the case of difunctional monomers the obtained enthalpy of polymerization is lower as compared to single double bond monomers. We obtained $43-44 \mathrm{~kJ} / \mathrm{mol}$ which is in the same range as those found by $\mathrm{H}$. Stenzenberger, ${ }^{1,9} 42 \mathrm{~kJ} / \mathrm{mol}$, and Fry and Lind, ${ }^{12}$ $45 \pm 3 \mathrm{~kJ} / \mathrm{mol}$. To be sure that the reaction was complete, after running the first scan to $380^{\circ} \mathrm{C}$, we cooled the sample to room temperature and repeated the DSC measurement. No residual exotherm was observed during this second scan. Fry and Lind ${ }^{12}$ have performed a similar experiment, but they have also measured the double bond conversion after the first scan with the help of ${ }^{13} \mathrm{C}-\mathrm{CP}$ Mas-nuclear magnetic resonance (NMR). They found the value $x=0.84$ indicating that residual double bonds are buried in the BMI network and cannot polymerize during the first and second DSC scans. Fourier transform infrared (FTIR) analysis of our cured samples (BDM as well as BT) also led to a value in this range.

Taking into account $\mathrm{x}=0.84$, the reaction enthalpy for $\mathrm{BMI}$ can be estimated to be in the range of $\Delta H_{p} \sim 52 \mathrm{~kJ} / \mathrm{mol}$ (mole of double bonds). This is still much lower than that for the monofunctional monomer. This low value can be explained as a result of some steric hindrance.

To verify this assumption, mixtures of mono and difunctional monomers ( $N$-PMI and $\mathrm{BMI}$ ) have been studied. The reaction enthalpy is constant, $85 \pm 3 \mathrm{~kJ} / \mathrm{mol}$, from 0 to $25 \%$ by weight of $\mathrm{BMI}$; then it decreases rapidly to $62 \pm 3 \mathrm{~kJ} / \mathrm{mol}$ for $30 \%$ b.w.; and then to $45 \pm 3 \mathrm{~kJ} / \mathrm{mol}$ for pure difunctional monomers. In Figure 2 this behavior is compared to the increase in $T_{g}$ with the amount of $\mathrm{BMI}$ of the final networks, and to the decrease in $\Delta C_{p}$ at $T_{g}$ (both difunctional monomers lead to almost exactly the same curves). The $T_{g}$ of pure $N$-PMI is $140^{\circ} \mathrm{C}$ with $\Delta C_{p} \sim 0.3 \mathrm{~J} . \mathrm{g} / \mathrm{K}$, but $\Delta C_{p}$ decreases drastically when the amount of difunctional monomer increases, and is less than $0.05 \mathrm{~J} . \mathrm{g} / \mathrm{K}$ for $30 \% \mathrm{BMI} \mathrm{b.w..} \mathrm{For}$ higher amounts of $\mathrm{BMI}$ it is not possible to detect the $T_{g}$ of the network. $\Delta C_{p}$ is related to the chain mobility, and it is clear that the low values for the heat of polymerization of $\mathrm{BMI}, \Delta H_{p}$, correspond to a very low mobility of the polymeric chains.

Table II. Maximum temperatures, heats of polymerization and activation energies as determined from DSC analysis for the polymerization of BDM/BT ( $70 / 30$ by weight) using various amounts of imidazole or benzimidazole

\begin{tabular}{|ll|c|c|c|c|}
\hline Initiator & & {$[\mathrm{I}] /[\mathrm{M}] \mathrm{mol} \%$} & $\boldsymbol{T}_{p}\left({ }^{\circ} \mathbf{C}\right)^{\mathrm{a}}$ & $\Delta \mathbf{H}_{p}(\mathbf{k J} / \mathbf{m o l})^{\mathbf{b}}$ & $\boldsymbol{E}_{a}(\mathbf{k J} / \mathbf{m o l})$ \\
\hline None & - & 0 & 240 & 50.7 & 115 \\
Imidazole & 0.1\% b.w. & 0.5 & 222 & 45 & 88 \\
& 0.2\% b.w. & 1.0 & 210 & 23 & 72 \\
Benzimidazole & 0.1\% b.w. & 0.3 & 247 & 41 & Not determined \\
& 1\% b.w. & 2.8 & 220 & 37 & \\
& 5\% b.w. & 14.2 & 205 & 33 & \\
\hline
\end{tabular}

\footnotetext{
${ }^{\mathrm{a}} \mathrm{q}=10 \mathrm{~K} / \mathrm{min}$.

${ }^{\mathrm{b}}$ Relative to a single double bond.
} 


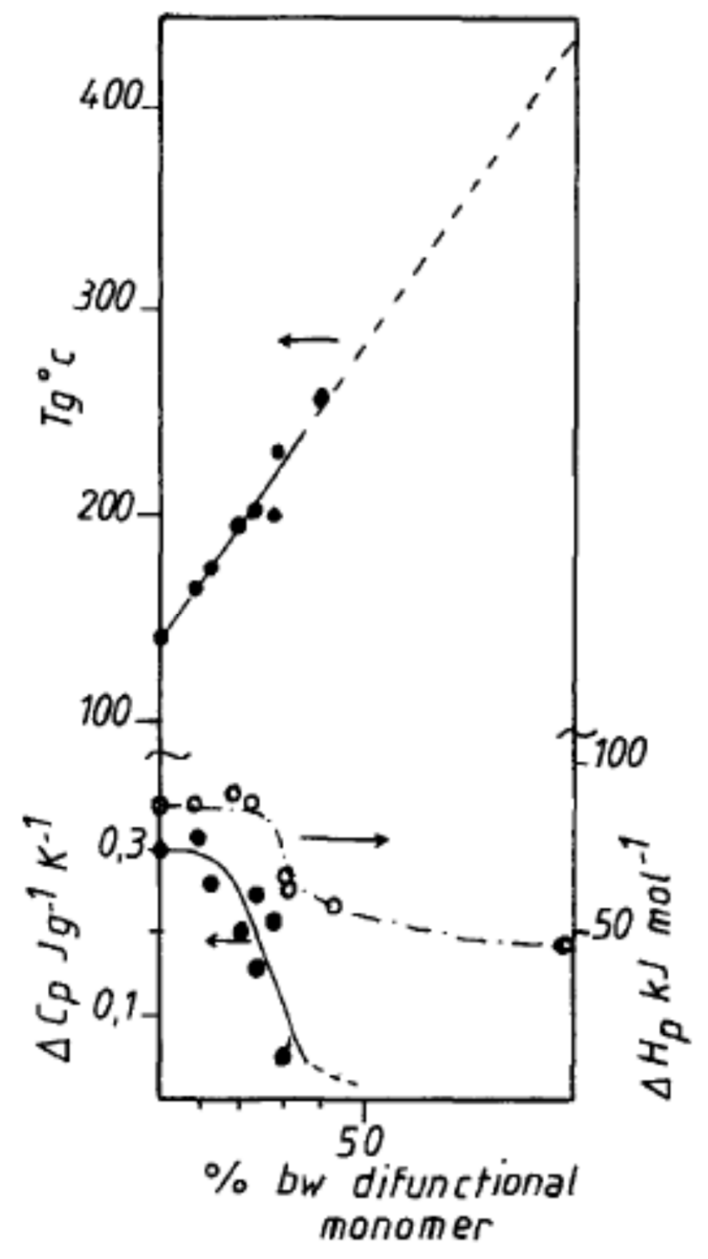

Figure 2. Comparison between the enthalpy of polymerization, $\Delta H_{p}$, per mole of double bonds with the glass transition temperature and the mobility $\left(\Delta C_{p}\right.$ at $\left.T_{g}\right)$ of the final networks based on blends of $\boldsymbol{N}$-PMI and BDM (or BT).

Calorimetric measurements have also been performed to obtain information about the influence of initiators like imidazole or benzimidazole. Experiments were run with the eutectic mixture BDM/BT, 70-30 b.w., to determine its influence on the different parameters. The main results are reported in Table II.

The addition of imidazole or benzimidazole had two effects: first the temperature of the exotherm peak maximum and the activation energy were lowered, as one might expect from an initiator. This effect was all the more pronounced as the amount of initiator used was increased. But curiously, the use of imidazole in increasing amounts also led to decreasing values for the heat of polymerization, $\Delta H_{p}$. We did not observe such a behavior for the monofunctional compound $\mathrm{N}$-phenylmaleimide; ${ }^{13}$ in this case, the addition of imidazole lowered $T_{p}$ as well as the activation energy but did not modify $\Delta H_{p}$ (85 kJ/mol for $N$-phenylmaleimide). This stated, it is difficult to account for this dependence of $\Delta H_{p}$ on the amount of imidazole, since if we refer, for example, to a possible transfer to the initiator, a similar mechanism should occur in the polymerization of the monofunctional compound. Residual double bonds were not measured after the first DSC scan for the BMI polymerization.

Table II also shows that benzimidazole was less efficient as an initiator than imidazole. 


\section{Isothermal Reactions: Kinetics and Gelation}

The kinetics of several isothermal polymerizations was investigated by size exclusion chromatography (SEC), and by viscosity and viscoelastic measurements. Gelation is defined as the critical point at which the curing system yields indefinitely large polymer structures or macrostructures which extend throughout the volume of the system. At the gel point the curing system suddenly transforms from a viscous liquid to an elastic gel. The gelation time may be determined as:

1. The time $t_{\text {ins }}$, when the sample starts to be partially insoluble in a strong solvent. For the BMI samples we have chosen the solvent dimethylformamide (DMF) instead of the classical tetrahydrofuran (THF).

2. The time $t_{\eta}$ when the viscosity tends to infinity. However, it is not practical to have an "infinite" viscosity and by convention we take the time when the viscosity equals $5.10^{4}$ Pa.s.

3. The time $t_{G^{\prime}=G^{\prime \prime}}$ at which the elastic and viscous moduli $G^{\prime}$ and $G^{\prime \prime}$ are equal. ${ }^{14,15}$ Measurements have been taken at a high frequency, $100 \mathrm{rad} / \mathrm{s}$. If the reaction is not too fast, it is easy to determine this point. An example is given in Figure 3. But in fact this point is frequency dependent, and recent results have shown that it is more correct to take the point at which $\tan \delta$ is independent of the frequency. In the case of epoxy systems this point is generally obtained for $\tan \delta$ higher than 1 . For example the point independent of the frequency is obtained for $\tan \delta=1.3$ for an epoxy system with a cycloaliphatic diamine curing agent. ${ }^{16}$ But on the time scale the difference between $\tan \delta=1$ and $\tan \delta=1.3$ is small.

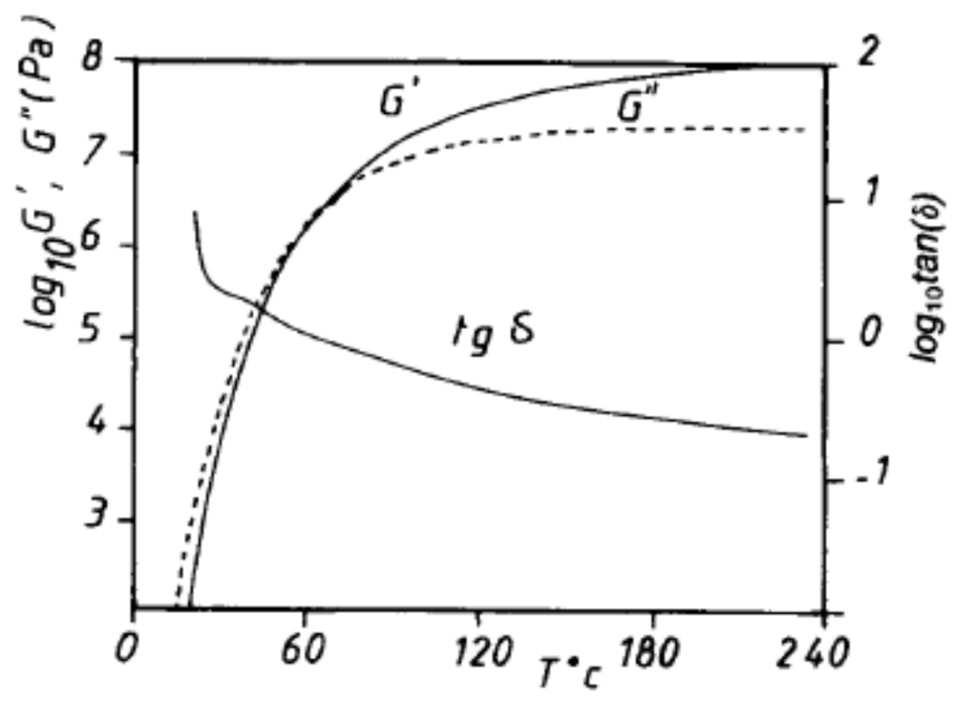

Figure 3. Example of viscoelastic measurements (100 rad/s) for the BDM/BT system at $T_{i}=150^{\circ} \mathrm{C}$, with $0.5 \%$ mol of initiator. 


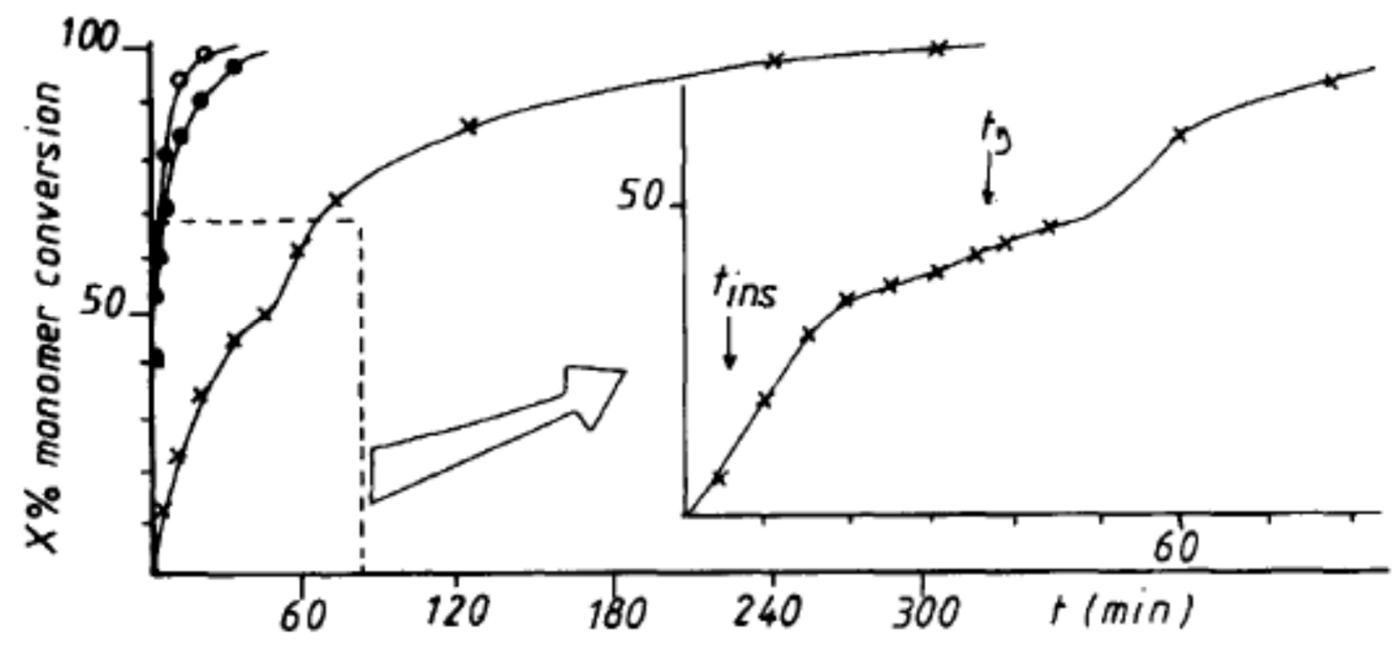

Figure 4. Monomer conversion, $X$, as a function of time for the polymerization of BDM/BT at $200^{\circ} \mathrm{C}$ and with different $\mathrm{mol} \%$ of initiator I $(x) 0 \%(0) 0.5 \%(O) 1 \%$.

Isothermal polymerizations were carried out using different percentages of imidazole at various temperatures. The monomer conversion, $X$, may be monitored as a function of time by following the decrease in the monomer peak height, $t$, in SEC chromatograms, in THF (on the soluble fraction of the samples) :

$$
X=1-h_{t} / h_{0}
$$

Both monomers BDM and $\mathrm{BT}$ are consumed at the same rate. Typical curves, which were obtained from the polymerization of $\mathrm{BDM} / \mathrm{BT}$ mixtures at $200^{\circ} \mathrm{C}$, are depicted in Figure 4 . According to the DSC scans results, a small quantity of imidazole, $0.5 \mathrm{~mol} \%$ (or $0.1 \%$ b.w.), increases drastically the polymerization rate.

For the thermal polymerization $(0 \% \mathrm{I})$ the beginning of the reaction is reported on a larger scale. Arrows indicate the gelation times $t_{\text {ins }}$ and $t_{\eta}$ found by SEC and viscosity measurements for these systems. Gel times are also reported in Table III. One remarkable fact is that an insoluble fraction appeared well before the increase in viscosity, $t_{\text {ins }} \ll t_{\eta}$, during thermal polymerizations. At $200^{\circ} \mathrm{C}$, monomer conversions are estimated from Figure 4 to be $X \sim 0.07$ at $t_{\text {ins, }}$ and $X \sim 0.45$ at $t_{\eta}$.

Statistical parameters of the pre- and post-gel stages may be obtained using different approaches ${ }^{17-20}$ derived from the pioneering work of Flory ${ }^{21}$ and Stockmayer. ${ }^{22}$ The main assumptions of the FloryStockmayer (FS) treatment are: (1) equal reactivities of the functionalities of each species; (2) absence of substitution effects (i.e., the reaction of a particular site does not alter the reactivity of the remaining unreacted sites); (3) absence of cyclic molecules in finite species. These conditions define an ideal polymerization, and in the case of an ideal chain-growth polymerization, the conversion at the gel point is very low, $x \leq 0.01$.

For a free-radical crosslinking copolymerization of monovinyl-divinyl systems, cyclization or intramolecular reactions have been found to be an intrinsic property. The consequence was that many experimental gelation results ${ }^{23-25}$ seemed to greatly deviate from the theoretical prediction by classical FS theory. Dusek et al ${ }^{26}$ proposed that, in the early stage of reaction the occurrence of cyclization forms internally crosslinked insoluble and rigid structures in the primary polymer chains referred to as "microgels". Many pendent vinyl groups are buried inside the microgels and show a reduced, or 
completely suppressed, reactivity. Then when the polymerization goes on, the microgel concentration continuously increases, and when the intermicrogel crosslinking dominate the reaction, the viscosity rises up very rapidly until the gel point, called in this case macrogelation. If we assume this gelation mechanism for the thermal polymerization of $\mathrm{BMI}, t_{\text {ins }}$ corresponds to the microgel formation and $t_{\eta}$ to macrogelation.

Table III. Influence of the initiator concentration on the gelation time, as measured with different techniques for the BDM/BT polymerization at $200^{\circ} \mathrm{C}$

\begin{tabular}{|c|c|c|c|}
\hline & \multicolumn{3}{|c|}{ Time $(\min )$} \\
\hline$\%$ mol I & $t_{\text {ins }}(\mathrm{DMF})$ & $t_{G^{\prime}=G^{\prime \prime}}$ & $t_{\eta}$ \\
\hline 0 & 5 & - & 36 \\
0.5 & 3 & 5 & 4 \\
1.0 & 2 & - & (not measurable) \\
\hline
\end{tabular}

This may be the result of numerous cyclization reactions as well as of a decrease in the reactivity of the double bonds, both by a substitution effect and because some of them get trapped inside the microgels. In Figure 4, one can notice that the appearance of insoluble microgels had no immediate influence on the reaction kinetics. A decrease in the rate of conversion was observed but somewhat after, at $t \approx 20 \mathrm{~min}$. This decrease became more pronounced after the system became very viscous, which would agree with a now partly diffusion-controlled reaction or with a lower radical concentration. However, the conversion increased at an even higher rate again after a short while. The overall behavior is difficult to explain. It is easy to explain the decrease in the polymerization rate when the viscosity increases, using a diffusion effect argument. However, it is more difficult to understand why the polymerization rate increases again $10 \mathrm{~min}$ after macrogelation. It is possible to refer to the Trommsdorff effect: the increase in viscosity lowers the rates of the reactions involving two polymeric molecules (termination and transfer to the polymer) much more than the rates of the reactions involving at least one small molecule (initiation and propagation) and thus the overall polymerization rate increases tremendously.

\section{Anionic Isothermal Reactions: Influence of Temperature and Initiator Concentration}

Imidazole drastically increases the polymerization rate (Table II and Fig. 4). The micro- and macrogelation times at a high isothermal curing temperature, $T_{i}=200^{\circ} \mathrm{C}$, are compared in Table III. There is a large difference between the values in the case of thermal polymerization, but the results are in the range of experimental errors in the case of polymerizations initiated by imidazole. For this reason, experiments have been performed at a lower temperature, $150^{\circ} \mathrm{C}$.

SEC experiments give the monomer conversion, $X$. If we assume that all the double bonds are equally reactive (i.e., if there is no substitution effect), the conversion of the vinyl functions, $x$, may be deduced from $X$ by the expression:

$$
1-X=(1-x)^{2}
$$


since 1-X describes the probability of finding a nonreacted BMI molecule and thus of finding simultaneously two nonreacted vinyl functions. Using this assumption we find:

$$
x_{\mathrm{SEC}}=1-\left(h_{t} / h_{0}\right)^{1 / 2}
$$

The evolution of the double-bond conversion, $x$, is displayed in Figure 5. $x$ was determined from SEC measurements $\left(x_{S E C}\right)$ but also chemically by reacting the remaining double bonds with morpholine.

Microgelation occurs at $t_{\text {ins }}=50 \mathrm{~min}$, which is before macrogelation, $t_{G^{\prime}=G^{\prime \prime}}=60 \mathrm{~min}$ or $t_{\eta}=70 \mathrm{~min}$. Both curves of $x$ versus $t$ are parallel up to macrogelation; if eq. (4) is correct, all the double bonds are equally reactive before gelation. After gelation the two curves are different: gelation appears on the $x$ conversion curve as a decrease in the polymerization rate. On the $x_{\mathrm{SEC}}$ curve, as shown in Figure 4 , the polymerization rate starts decreasing, then reaccelerates $10 \mathrm{~min}$ after gelation, and finally returns to its former value (both curves are parallel in the end). Note that here the mechanism is anionic and thus the Trommsdorff effect cannot be encountered.

The different behaviors observed on the two conversion curves indicate that after macrogelation there is a strong substitution effect: once the first double bond of a monomer molecule has reacted, the reactivity of the second one becomes very low. A consequence of this substitution effect is that the monomer conversion increases rapidly after macrogelation, and thus explains the form of the curves $x$ versus $t$ in Figure 4 and $x_{\text {SEC }}$ versus $t$ in Figure 5 .

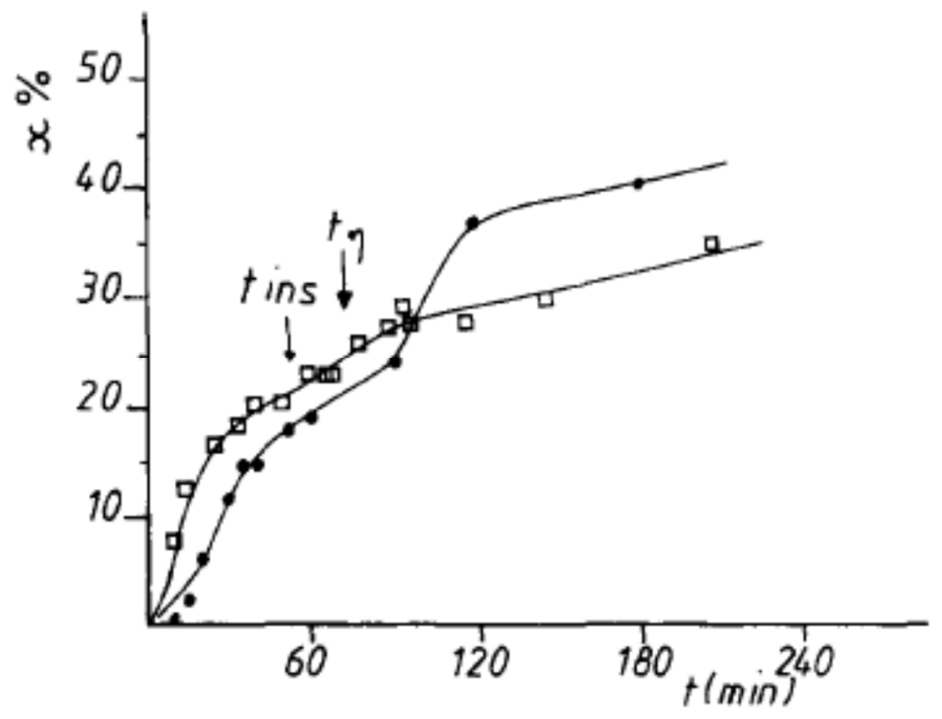

Figure 5. Double bond conversion, $x$, as a function of time for the polymerization of $B D M / B T$ at $150^{\circ} \mathrm{C}$ ( $0.5 \mathrm{~mol} \%$ imidazole) (O) SEC measurements, $x_{\text {SEC }}(\square)$ chemical determination, $x$.

The results obtained for the gelation times at various isothermal curing temperatures, $T_{i}$, are displayed in Table IV. In the case of anionic polymerization the different criteria lead to quite close gelation times.

Boots $^{27}$ has shown using a simulation, that the degree of heterogeneity of a network during polymerization depends on the ratio of the initiating rate, $R_{i}$, to the propagating rate, $R_{p}$. When the ratio is equal to 1 (case of a step-growth polymerization) the process of the network formation is homogeneous. When this ratio is high, corresponding to a very low $R_{i}$ compared to $R_{p}$, the process of the network formation is heterogeneous. Thus we can say that the anionic polymerization of BMI is 
much more "homogeneous" than when it is thermally initiated, which is possibly a result of a faster initiation step.

If this is so, the ratio

$$
p=\frac{R_{p}}{R_{p}+R_{i}}=\frac{R_{p}}{R_{p}+R_{t}+R_{t r}}
$$

(where $R_{i}, R_{p}, R_{t}$, and $R_{t r}$ are the rates of initiation, propagation, termination, and transfer, respectively) should be less than unity. This is in good agreement with the higher value found for $x_{\text {ins }}$ in the case of anionic polymerization $(x \sim 0.2)$ as compared to the thermal polymerization for a tetrafunctional monomer, since $x_{\text {gel }}$ depends on the parameter, ${ }^{21-23}$

$$
x_{g e l}=\frac{1-p}{2 p}
$$

Table IV. Gelation times associated with the polymerization of BDM/BT (0.5 mol \% imidazole) at various temperatures $T_{i}$

\begin{tabular}{|c|c|c|c|c|}
\hline$T_{i}\left({ }^{\circ} \mathrm{C}\right)$ & $t_{\text {ins }}(\min )$ & $t_{G^{\prime}=G^{\prime \prime}}(\min )$ & $t_{\eta}(\min )$ & $x \%$ at $t_{\text {ins }}$ \\
\hline 140 & 180 & - & - & 28 \\
150 & 50 & 60 & 70 & 20 \\
155 & 40 & - & - & 24 \\
160 & 30 & 30 & 31 & 27 \\
170 & 20 & - & 19 & 26 \\
180 & 10 & - & 9 & 22 \\
190 & 5 & - & 7 & 16 \\
200 & 3 & 5 & 4 & 16 \\
\hline
\end{tabular}

$x$ is determined from SEC measurements ( $x_{\mathrm{SEC}}$ ) except for $T_{i}=150^{\circ} \mathrm{C}$ (see Fig. 5).

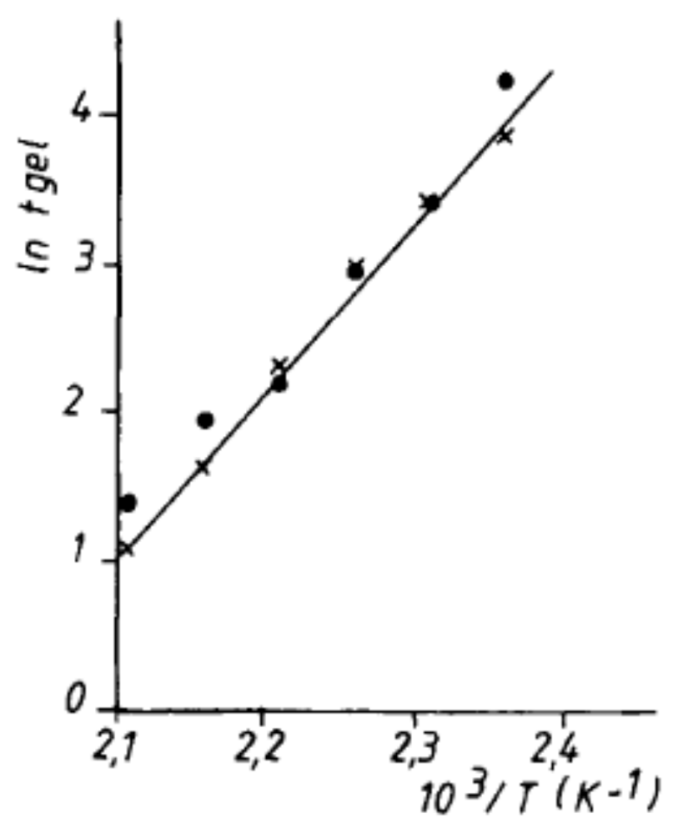

Figure 6. Arrhenius plot for $t_{\text {ins }}(x)$ and $t_{\eta}(0)$ for BDM/ BT (70/30) polymerization initiated by $0.5 \mathrm{~mol} \%$ imidazole. 
The values given in Table IV indicate that the gelation times become shorter and shorter as the reaction temperature increases. More precisely, in the case of anionic polymerization, the plot of $\ln t_{\text {ins }}$ or $\ln t_{\eta}$ versus $1 / T$ leads to a straight line as shown in Figure 6 . The same mechanism should thus be involved at every temperature, and consequently $x_{\text {gel }}$ should be independent of the temperatures. This is roughly the case if we refer to Table IV: all the gel conversions are equal to $22 \pm 6 \%$.

This is true up to a cure temperature of $190^{\circ} \mathrm{C}$. However, one must keep in mind that the maximum of the exotherm corresponding to the thermally induced polymerization of BDM/BT (70/30) was observed at $240^{\circ} \mathrm{C}$. A radical mechanism is thus likely to interfere with the anionic mechanism at temperatures higher than $190^{\circ} \mathrm{C}$.

If we assume that the mechanism is the same at all temperatures and that $x_{\mathrm{gel}}$ is constant, then an activation energy may be deduced from the Arrhenius plot displayed in Figure 6: here we find $E_{a}=94 \mathrm{~kJ} / \mathrm{mol}$, instead of $115 \mathrm{~kJ} / \mathrm{mol}$ for the thermally initiated system. The same activation energy determined from DSC measurements (Kissinger's treatment) for the polymerization of BDM/BT (70/30) initiated by $0.5 \mathrm{~mol} \%$ imidazole was equal to $88 \mathrm{~kJ} / \mathrm{mol}$. Both methods thus give consistent results.

Table V. Comparison of the values of $t_{\text {ins }}$ and $x_{\text {gel }}$ found for the curing of BDM/BT (70/30) at various temperatures, using either $0.5 \mathrm{~mol} \%$ or $1 \mathrm{~mol} \%$ of imidazole as the initiator; $x$ is determined from SEC measurements

\begin{tabular}{|c|c|c|c|c|}
\hline & \multicolumn{2}{|c|}{$t_{\text {ins }}(\mathrm{min})$} & \multicolumn{2}{c|}{$x_{\text {gel }}(\%)$} \\
\hline$T_{i}\left({ }^{\circ} \mathrm{C}\right)$ & $\begin{array}{l}0.5 \mathrm{~mol} \% \\
\text { imidazole }\end{array}$ & $\begin{array}{l}1.0 \mathrm{~mol} \% \\
\text { imidazole }\end{array}$ & $\begin{array}{l}0.5 \mathrm{~mol} \% \\
\text { imidazole }\end{array}$ & $\begin{array}{c}1.0 \mathrm{~mol} \% \\
\text { imidazole }\end{array}$ \\
\hline 150 & 50 & 40 & 20 & 33 \\
160 & 30 & 20 & 27 & 30 \\
170 & 20 & 10 & 26 & 43 \\
180 & 10 & 6 & 22 & 31 \\
190 & 5 & 3 & 16 & 42 \\
200 & 3 & 2 & 16 & 29 \\
\hline
\end{tabular}

The same kind of kinetic measurements as reported previously were run for the curing of BDM /BT (70/30) using $1.0 \mathrm{~mol} \%$ imidazole instead of $0.5 \mathrm{~mol} \%$. The results obtained are listed in Table $\mathrm{V}$. The increase in the amount of initiator had two effects: at first, it lowered the gelation time, whatever curing temperature was used; this is quite logical. The activation energy calculated from DSC measurements is equal to $72 \mathrm{~kJ} / \mathrm{mol}$, i.e., less than the value of $88 \mathrm{~kJ} / \mathrm{mol}$ found for $0.5 \mathrm{~mol} \%$ initiator.

But using double the amount of initiator also led to much higher conversions at the gel point. Here the mean value of $x_{\text {gel }}$ is equal to $35 \%$, instead of $22 \%$ for $0.5 \mathrm{~mol} \%$ imidazole. This is compatible with a shorter gelation time if we assume that the initiation rate is greatly enhanced: the ratio $p=\frac{R_{p}}{R_{p}+R_{i}}$ decreases and thus the value of $x_{g e l}=\frac{1-p}{2 p}$ increases accordingly. 


\section{Vitrification phenomena}

In the curing of thermosets, the variations in the glass transition temperature $T_{g}$ are important to follow since the occurrence of vitrification can have a great influence on the overall kinetics. Epoxy systems often become diffusion-controlled after $t_{\text {vit, }}$ the time for which the increasing $T_{g}$ reaches the value of the curing temperature $T_{i .}{ }^{28,29}$ Moreover, $T_{g}$ is a parameter characteristic of the structure of the curing network at a particular stage, and, if the chemical phenomena are always the same, $T_{g}$ should be linked to $x$ by a unique relation depending only on the mechanism but not on $T_{i .}{ }^{30}$

Here the $T_{g}$ was monitored as a function of time for the curing of BDM/BT (70/30 b.w.; $0.5 \mathrm{~mol} \%$ imidazole) at various temperatures. For a very high curing temperature such as $200^{\circ} \mathrm{C}$, values of $T_{g}$ could not be obtained from DSC measurements because the heat capacity changes were too small, due to a higher crosslinking density (see Fig. 2). In this case, the $T_{g}$ was deduced from viscoelastic measurements: in the first step, the sample was cured isothermally for a given time in the viscoelasticimeter. The specimen was then quenched at a temperature below the $T_{g}$ and $G^{\prime}, G^{\prime \prime}$ and $\tan \delta$ were then monitored as a function of temperature using a heating rate of $10 \mathrm{~K} / \mathrm{min}$. If we plot $G^{\prime}$ vs. $T$ (as represented in Fig. 7), we observe at first a decrease in $G^{\prime}$ (corresponding to the glass transition). Then the elastic modulus increases again, since the network is being postcured: the remaining double bonds start reacting and the network becomes more densely crosslinked. In these experiments, $T_{g}$ was taken at the first inflection on the $G^{\prime}$ versus $T$ curves rather than at the maximum of the peak in $G^{\prime \prime}$ which is more characteristic of the post-cure reaction.

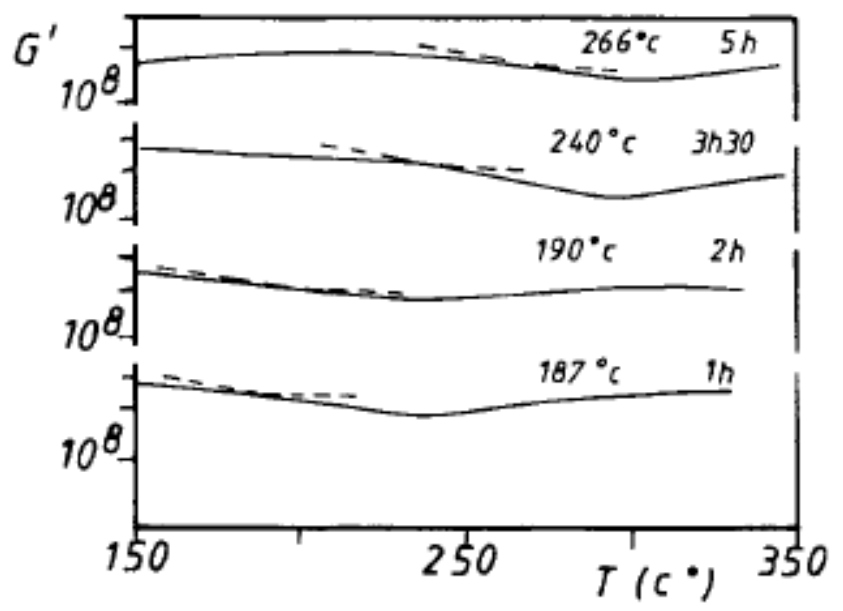

Figure 7. Elastic modulus $G^{\prime}$ versus temperature (heating rate: $10 \mathrm{~K} / \mathrm{min}$ ) for partially cured networks of BDM/BT $(70 / 30)$ and $0.5 \mathrm{~mol} \%$ imidazole $\left(t_{\text {cure }}=1 \mathrm{~h}, 2 \mathrm{~h}, 3.5 \mathrm{~h}\right.$ and $\left.5 \mathrm{~h} ; T_{i}=200^{\circ} \mathrm{C}\right)$.

The direct plot of $T_{g}$ versus curing time for various temperatures is depicted in Figure 8 . The variations of $T_{g}$ with time are quite similar to those observed for the conversion, except that the momentary "slow-down" in the increase in $T_{g}$ is less pronounced and occurs sometimes quite a long time after macrogelation (especially when the curing temperature gets higher). Moreover, for low values of $T_{i}$ the final plateau is reached well before $t_{\text {vit, }}$ as if the reaction becomes diffusion controlled long before vitrification. On the contrary, for $T_{i}=200^{\circ} \mathrm{C}, T_{g}$ keeps increasing rapidly after vitrification. The behavior 
observed for $T_{i}=170$ and $180^{\circ} \mathrm{C}$ is intermediate (i.e., the beginning of the plateau coincides roughly with vitrification).

The anomalous behavior encountered for $T_{i}=200^{\circ} \mathrm{C}$ cannot be explained only by the difference in the experimental techniques [viscoelasticimetry $\left(200^{\circ} \mathrm{C}\right.$ ) versus DSC (other temperatures)]. This should result in a shift in the transition temperatures but not in such a different slope after vitrification. It may be a consequence of the thermal initiation of the polymerization (or dimerization) starting to interfere with the anionic mechanism. Even if the mobility of the chains becomes very low after $t_{\text {vit, }}$ new radicals can be generated inside the network and give rise to more crosslinks, hence the increase in $T_{g}$. In any case, the variations in $T_{g}$ with $x$ cannot be the same as that observed for lower curing temperatures since there are now two mechanisms instead of one and thus it is not surprising that the general shape of the curve is not the same.

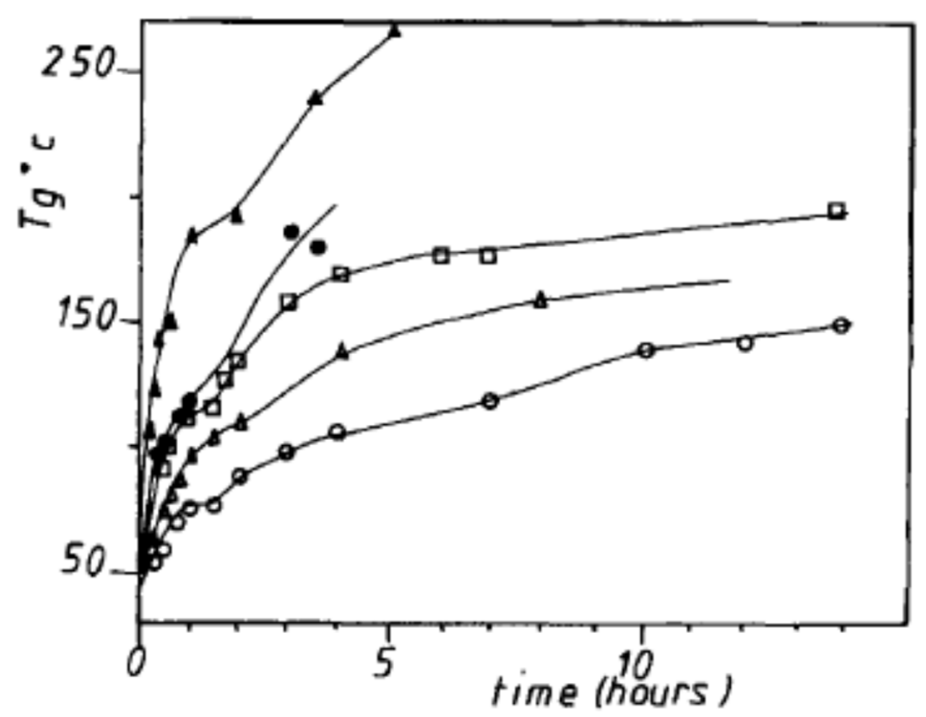

Figure 8. Glass transition temperature $T_{g}$ during the curing of BDM/BT (70/30) with $0.5 \mathrm{~mol} \%$ imidazole: $T_{i}=150^{\circ} \mathrm{C}(O) ; 160^{\circ} \mathrm{C}(\triangle) ; 170^{\circ} \mathrm{C}(\square) ; 180^{\circ} \mathrm{C}(O) ; 200^{\circ} \mathrm{C}(\triangle)$.

For the lower curing temperatures, the thermal initiation (and thus the radical mechanism of polymerization or dimerization ) is unlikely to occur. Since we are considering a very highly crosslinked network, at a lower $T_{i}\left(150,160^{\circ} \mathrm{C}\right)$ the motions of the reactants can be hindered very early in the reaction, i.e. at low conversions and for values of $T_{g}$ lower than $T_{i}$. Now if we assume that in this case $T_{g}$ is linked to $x$ by a unique relation, we can consider the part of the curves when the reaction is only kinetically controlled and one can neglect diffusion. In this case, Wisanrakkit and Gillham ${ }^{29}$ propose writing the kinetic equation as:

$$
\frac{d x}{d t}=k(T) \cdot f(x)
$$

where $k$ depends only on the temperature, and $f$ on the conversion $x$, thus on $T_{g}$. The former equation leads to the following expression:

$$
F\left(T_{g}\right)=\ln k(T)+\ln t
$$

For two given curing temperatures $T_{1}$ and $T_{2}$, let us denote $t_{1}$ and $t_{2}$ as the times corresponding to the same glass transition temperature, $T_{g}$. 


$$
\operatorname{lnk}\left(T_{1}\right)+\ln t_{1}=\ln k\left(T_{2}\right)+\ln t_{2}
$$

or

$$
\ln t_{2}-\ln t_{1}=\ln k\left(T_{1}\right)-\ln k\left(T_{2}\right)
$$

and thus $\ln t_{2}-\ln t_{1}$ is a constant (independent of time) which depends only on the curing temperatures. This means in fact that all the $T_{g}$ versus Int curves may be deduced from one another simply by shifting them along the $\ln t$ axis (as long as diffusion does not slow down the reaction).

If we plot $T_{g}$ versus Int in our case, we obtain the curves displayed in Figure 9. One sees that the lower parts of all the curves seem to be superposable $\left(T_{g}<130^{\circ} \mathrm{C}\right)$; i.e., a master curve which gives the general shape for the variation in $T_{g}$ with Int actually exists in this temperature range. (This would confirm the assumption made when noticing that the variation of $\operatorname{In} t_{\text {gel }}$ versus $1 / T$ gave a straight line: the mechanism of the reaction, up to the gel point, seems to be roughly the same at all the curing temperatures, $T_{i}$ ). In these conditions, Wisanrakkit and Gillham ${ }^{29}$ also define a shift factor, $A_{T}$, by choosing a reference curing temperature, $T_{0}$. Then for any curing temperature, $T$ :

$$
A_{T}=\ln t-\ln t_{0}=\ln k-\ln k_{0}=-\frac{E_{a}}{R}\left(\frac{1}{T}-\frac{1}{T_{0}}\right)
$$

where $E_{a}$ represents the single apparent activation energy of the reaction. If we try to apply this equation to our results, we can plot $A_{T}=\ln t-\ln t_{0}$ versus $(1 / T)$ choosing for example $T_{0}=150^{\circ} \mathrm{C}$ $(423 \mathrm{~K})$. The resulting diagram is shown in Figure 10. We obtain a straight line, whose slope yields a value of $E_{a}=97 \mathrm{~kJ} / \mathrm{mol}$. This is consistent with the values deduced from DSC scans using Kissinger's treatment $\left(E_{a}=88 \mathrm{~kJ} / \mathrm{mol}\right)$ and from the Arrhenius plot of In $t_{\text {gel }}$ versus $1 / T\left(E_{a}=94 \mathrm{~kJ} / \mathrm{mol}\right)$. One should keep in mind that this interpretation is valid only when diffusion has no influence on the reaction kinetics, i.e. on a modest part of some of our curves.

\section{Time-Temperature-Transformation Diagram}

After completing the study of the curing of BDM/BT initiated by $0.5 \mathrm{~mol} \%$ imidazole, it was possible to establish the time-temperature-transformation cure diagram which describes the system. ${ }^{31}$ This diagram is shown in Figure 11. $T_{g \infty}$ (corresponding to a conversion $x=100 \%$ ) was extrapolated to about $400^{\circ} \mathrm{C}$ from DSC measurements using decreasing amounts of the monofunctional compound $\mathrm{N}$-PMI (see Fig. 2). $T_{g 0}$ corresponds to the uncured system $\left(T_{g 0}=43^{\circ} \mathrm{C}\right)$.

The parameter ${ }_{\text {gel }} T_{g}$ is a characteristic of the system. It indicates the curing time and temperature for which gelation and vitrification occur simultaneously.

If we assume that a single mechanism is involved in the cure of this system, $x_{\text {gel }}$ must be a constant, independent of the temperature; this is roughly what we found. If $T_{g}$ is linked to $x$ by a unique relation, then the value of $T_{g}$ at the gel point must also be a constant independent of the curing temperature. In particular, this constant value of $T_{g}$ (gel) should be equal to gel $T_{g}$. Values of $T_{g}$ at the gel point were thus measured for the various curing temperatures and are reported in Table VI. There is little dispersion of the values, and they are centered on a mean value of $72^{\circ} \mathrm{C}$, which was estimated to be the actual ${ }_{\text {gel }} T_{g}$. When applied to the cure of BDM/BT with $1.0 \mathrm{~mol} \%$ imidazole, the same method led to a slightly higher value of gel $_{g}=76^{\circ} \mathrm{C}$. 


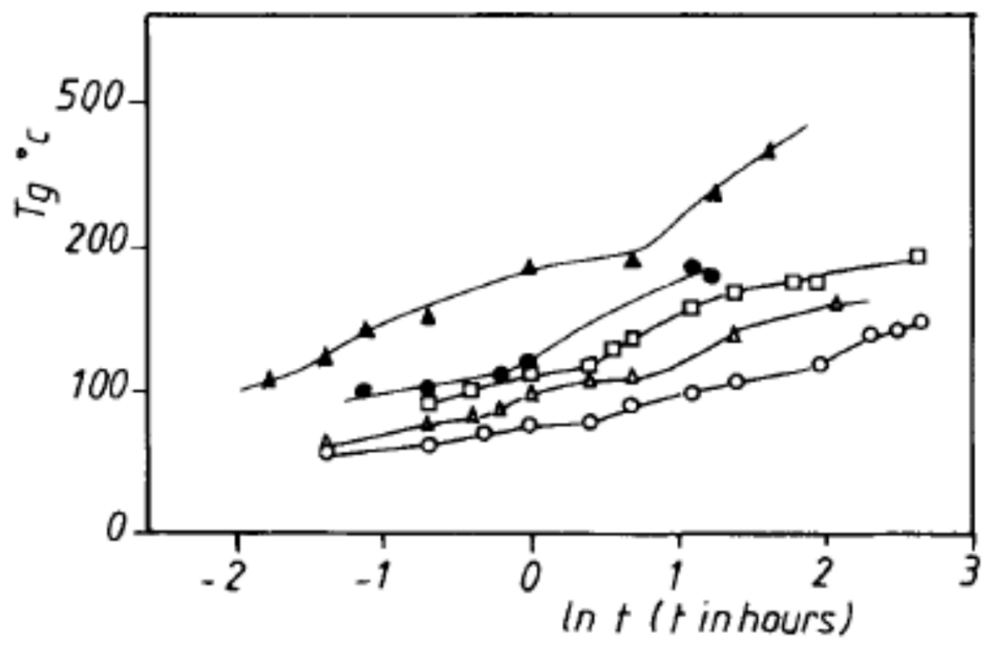

Figure 9. Evolution of the glass transition temperature $T_{g}$ with Int for the curing of BDM/BT (70/30) with $0.5 \mathrm{~mol} \%$ imidazole at various temperatures: $T_{i}=150^{\circ} \mathrm{C}(O) ; 160^{\circ} \mathrm{C}(\triangle) ; 170^{\circ} \mathrm{C}(\square) ; 180^{\circ} \mathrm{C}(O)$; $200^{\circ} \mathrm{C}(\Delta)$.

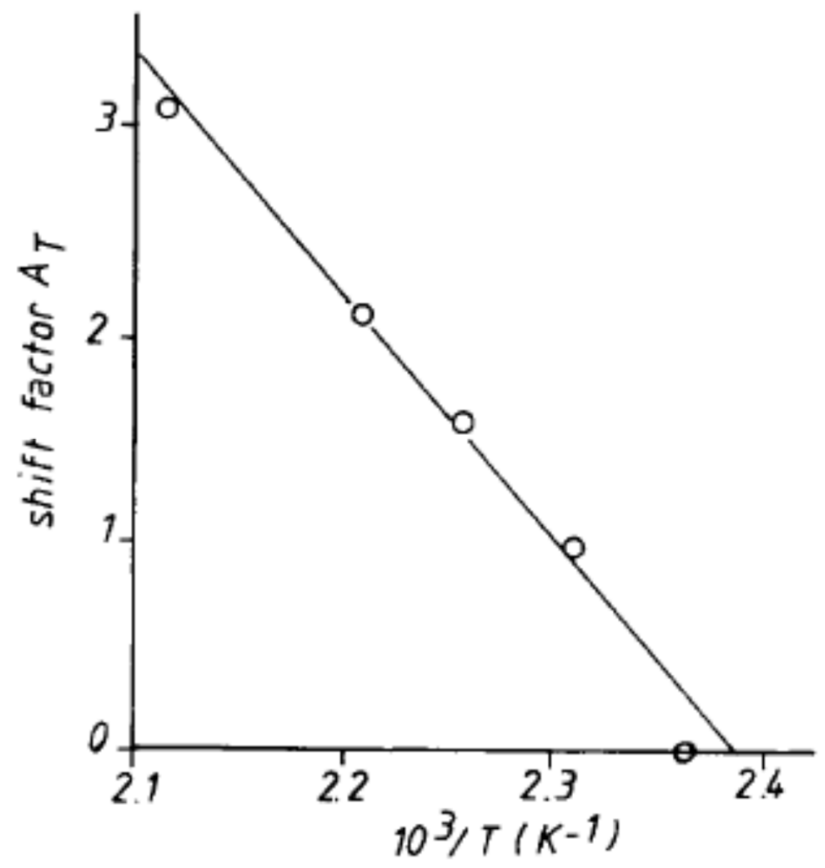

Figure 10. Arrhenius plot of the shift factors $A_{T}=\operatorname{lnt}-\ln t_{150^{\circ} \mathrm{C}}$, deduced from Figure 9, vs. $1 / T$. 


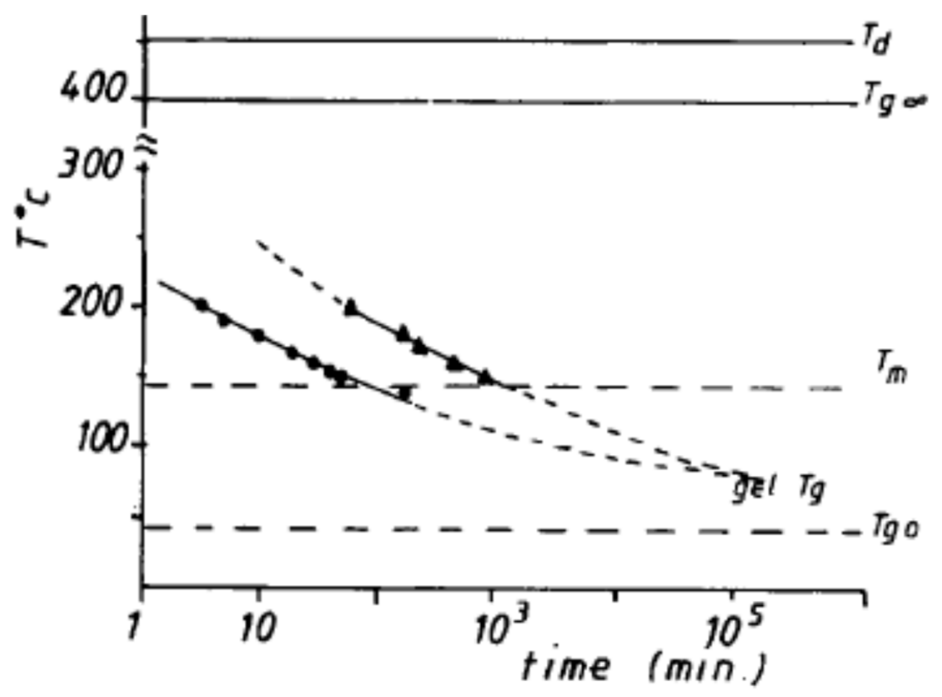

Figure 11. Time-temperature-transformation cure diagram of the system BDM/BT (70/30) $0.5 \mathrm{~mol} \%$ imidazole ( $\boldsymbol{\Delta}$ : vitrification; $\bullet$ : gelation).

Table VI. Glass transition temperatures at the gel point for the cure of BDM/BT (70/30) with 0.5 or $1.0 \mathrm{~mol} \%$ imidazole at various temperatures $T_{i}$

\begin{tabular}{|c|c|c|}
\hline & \multicolumn{2}{|c|}{$T_{\mathrm{g}}(\mathrm{gel})\left({ }^{\circ} \mathrm{C}\right)$} \\
\hline$T_{i}\left({ }^{\circ} \mathrm{C}\right)$ & $0.5 \mathrm{~mol} \% \mathrm{I}$ & $1.0 \mathrm{~mol} \% \mathrm{I}$ \\
\hline 150 & 71 & 79 \\
160 & 70 & 75 \\
170 & 77 & 79 \\
180 & 78 & 77 \\
190 & 68 & 76 \\
200 & 66 & 73 \\
\hline
\end{tabular}

\section{CONCLUSION}

Bismaleimide prepolymers are easily cured to produce highly thermostable networks. In this work, we tried to acquire a more precise idea of how the reaction proceeds, whether it is anionically or simply thermally induced, and how both mechanisms may interfere with each other. We are now able to provide a general scheme for this polymerization, although finer experiments would be necessary to make a perfect description of the system.

It appears that the thermally initiated polymerization proceeds in a rather heterogeneous manner: gelation is reached very rapidly but results at first only in the formation of microgels. The macrogelation of the system is sometimes observed much later, as the reaction proceeds. The heats of polymerization have been found to be quite close to the data available in the literature, although the extent of non-reacted functions is sometimes difficult to estimate. 
The use of imidazole or benzimidazole as anionic initiators allows the curing of bismaleimides at lower temperatures. The complete study of the system BDM/BT (70/30 b.w.)/0.5 mol \% imidazole gave us some insight into the polymerization mechanism which seems much more "homogeneous" than the thermally initiated mechanism; this is probably due to a faster initiation (as compared with the propagation). In the case of this particular system, several experimental observations agree confirming that there is only one mechanism, at least up to $190^{\circ} \mathrm{C}$, no matter what curing temperature is used; but diffusion may start to limit the kinetics at low conversions, i.e. well before vitrification. However, the conversion at the gel point is roughly constant and higher than that observed for the radical polymerization. By combining all the measurements, we were able to trace the general shape of the time-temperature-transformation cure diagram which totally depicts the behavior of BDM/BT/0.5 mol $\%$ imidazole. Some information about the cure initiated by $1 \mathrm{~mol} \%$ initiator was also obtained.

An important feature of this polymerization is that the reactivity of the second double bond of a monomer molecule is greatly reduced after gelation.

It is apparently difficult to reach very high conversions, and the nature of the last stages of the reaction (particularly termination) needs to be characterized more precisely. This will be very important to understand the relations between structure and properties of the final networks.

We wish to thank Rhône-Poulenc Co. for their financial and experimental support.

\section{REFERENCES}

1. H. Stenzenberger, in Structural Adhesives, A. J. Kinloch, Ed., Elsevier, London, 1986, p. 77.

2. I. K. Varma, S. P. Gupta, and D. S. Varma, J. Appl. Polym. Sci., 33, 151 (1987).

3. G. T. Kwiatkowski, L. M. Robeson, G. L. Brode, and A. W. Bedwin, J. Polym. Sci. Chem., 13, 961 (1975).

4. M. Acevedo, J. de Abajo, and J. G. de la Campa, Polymer, 31, 1955 (1990).

5. H. D. Stenzenberger, M. Herzog, W. Romer, R. Scheiblich, S. Pierce, and M. Canning, $30^{\text {th }}$ Nat. SAMPE Symp., 30, 1568 (1985).

6. H. E. Kissinger, Anal. Chem., 29(11), 1702 (1957).

7. S. Sourour and M. R. Kamal, Thermochim. Acta, 14, 41 (1976).

8. J. Galy, A. Sabra, J. P. Pascault, Polym. Eng. Sci., 26(21), 1514 (1986).

9. H. D. Stenzenberger, Appl. Polym. Symp., 22, 77 (1973).

10. I. M. Brown and T. C. Sandreczki, Macromolecules, 23, 94 (1990).

11. R. M. Joshi and B. J. Zwolinski, in Vinyl Polymerization. Heats of Polymerization and their Structural and Mechanistic Implications, G. E. Ham, Ed., Marcel Dekker, New York, 1967, Vol. 1, Part I, Chap. 8.

12. C. G. Fry and A. C. Lind, New Polym. Mater., 2(3), 235 (1990).

13. A. Seris, F. Méchin, and J. P. Pascault, Sympol 90 Proceedings, 277, Paris, Sept. 9-13 (1990).

14. C. Y. M. Tung and P. J. Dynes, J. Appl. Polym. Sci., 27, 569 (1982).

15. F. Chambon and H. H. Winter, Polym. Bull., 13, 499 (1985).

16. L. Cahouet and M. Fève (to be published). 
17. M. Gordon, Proc. R. Soc. London, A:268, 240 (1962).

18. C. W. Macosko and D. R. Miller, Macromolecules, 9, 199 (1976).

19. D. R. Miller and C. W. Macosko, Macromolecules, 9, 206 ( 1976).

20. R. J. J. Williams, Macromolecules, 21, 2568 (1988).

21. P. J. Flory, J. Am. Chem. Soc., 63, 3083 (1941).

22. W. H. Stockmayer, J. Chem. Phys., 11, 45 (1943).

23. K. Dusek, in Developments in Polymerization.3, R. N. Howard, Ed., Applied Science Publishers, London, 1982, Chap. 4.

24. K. Ito, Y. Murase, and Y. Yamashita, J. Polym. Sci. Polym. Chem., 13, 87 (1975).

25. G. Hild and R. Okasha, (a) Makromol. Chem., 186, 93 (1985); b) ibid, 186, 389 (1985).

26. K. Dusek, H. Galina, and J. Mikes, Polym. Bull., 3, 19 (1980).

27. H. M. J. Boots, Physica, 147A, 90 (1987).

28. A. T. Di Benedetto, J. Polym. Sci. Polym. Phys., B25, 1949 (1987).

29. G. Wisanrakkit and J. K. Gillham, J. Appl. Polym. Sci., 41, 2885 (1990).

30. J. P. Pascault and R. J. J. Williams, J. Polym. Sci. Polym. Phys., B28, 85 (1990).

31. J. B. Enns and J. K. Gillham, J. Appl. Polym. Sci., 28, 2567 (1983). 